\title{
Pain control with paracetamol from a sustained release formulation and a standard release formulation after third molar surgery: a randomised controlled trial
}

\author{
P Coulthard, ' C M Hill, ${ }^{2}$ J W Frame, ${ }^{3}$ H Barry, ${ }^{4}$ B D Ridge, ${ }^{5}$ and T H Bacon, ${ }^{6}$
}

\begin{abstract}
Objective To compare the analgesic efficacy and safety of a sustained release (SR) paracetamol formulation (Panadol Extend) with a standard immediate release (IR) formulation (Panadol) after third molar surgery.

Design A multi-centre, double-blind, randomised clinical trial. Methods Patients received either a single oral dose of SR paracetamol or IR paracetamol for pain after the removal of at least one impacted third molar requiring bone removal under general anaesthesia. Post-operative pain and pain relief assessments were undertaken at time intervals up to 8 hours. Global assessments of effectiveness were made at 4 and 8 hours. Any adverse events were also recorded.

Results Of 627 randomised patients, 314 were treated with SR paracetamol and 313 with IR paracetamol. In the per protocol population at 4 hours, $35.1 \%$ of the 252 patients on SR paracetamol rated the study medication as very good or excellent compared with $27.7 \%$ of the 258 patients on IR paracetamol. There were few statistically significant differences among the secondary parameters but where they did occur they favoured SR paracetamol. Trends in favour of SR paracetamol were observed among the secondary parameters and these tended to emerge at the later time points. For example, while there was no statistically significant difference in time to re-medication between the treatment groups, the estimated time to re-medication was longer for patients treated with SR paracetamol ( $4 \mathrm{hr} 5 \mathrm{~min})$ compared with IR paracetamol ( $3 \mathrm{hr} 10 \mathrm{~min})$. The high rate of re-medication observed is consistent with that reported for
\end{abstract}

\footnotetext{
${ }^{1}$ Senior Lecturer and Consultant, Oral and Maxillofacial Surgery, University Dental Hospital of Manchester, Higher Cambridge Street, Manchester; ${ }^{2}$ Consultant, Oral and Maxillofacial Surgery, University Dental Hospital of Cardiff, Heath Park, Cardiff; ${ }^{3}$ Professor and Consultant, Oral and Maxillofacial Surgery, University of Birmingham School of Dentistry, St Chad's Queensway, Birmingham; ${ }^{4}$ Consultant and Senior Lecturer, Oral and Maxillofacial Surgery, Dublin Dental Hospital, Lincoln Place, Dublin, Ireland; ${ }^{5,6}$ GlaxoSmithKline

Consumer Healthcare, Research and Development, St George's Avenue, Weybridge

Correspondence to:Paul Coulthard, Head of Oral and Maxillofacial Surgery, University Dental Hospital of Manchester, Higher Cambridge Street, Manchester M15 6FH email:paul.coulthard@man.ac.uk

REFEREED PAPER

Received 12.12.00; Accepted 04.04.01

(C) British Dental Journal 2001; 191: 319-324
}

IR paracetamol using the post-operative dental pain model ${ }^{4,6}$. No difference was observed between the SR paracetamol and IR paracetamol treatment groups in distribution, incidence or severity of adverse events.

Conclusions SR paracetamol and IR paracetamol are clinically and statistically equivalent. While SR paracetamol and IR paracetamol were similar in terms of both onset of analgesia and peak analgesic effect, SR paracetamol had a longer duration of activity than IR paracetamol. The safety profiles of SR paracetamol and IR paracetamol were found to be very similar.

Paracetamol (acetaminophen) is a non-opioid analgesic with $P$ antipyretic properties, effective in relieving mild to moderate pain. ${ }^{1}$ The mechanism of action is not completely understood, although it is thought to act primarily through inhibition of prostaglandin synthetase in the central nervous system. ${ }^{2}$ Paracetamol is an important drug for the control of pain in dentistry and several studies have confirmed its effectiveness. ${ }^{3-6}$ It is commonly prescribed as well as being available for retail sale. It is of particular value when NSAIDS are contraindicated, perhaps by known hypersensitivity or a history of gastrointestinal ulceration or bleeding. ${ }^{7}$ Paracetamol is also the analgesic of choice to supplement NSAIDS when these are expected to be ineffective to control a patient's pain. ${ }^{8}$

Paracetamol, like many other analgesics, has a short half-life (around 2-3 hours) which necessitates frequent dosing. The UK recommended regimen is 500-1000 mg every 4-6 hours. ${ }^{9}$ However, it would be advantageous if the duration of action were longer so that fewer daily doses could maintain therapeutic plasma levels. This would improve patient convenience and compliance and be of benefit to the patient at night-time, for example. Panadol Extend is a sustained release (SR) product containing $665 \mathrm{mg}$ paracetamol which has been designed to give analgesia for up to 8 hours after dosing. The tablet has a bi-layer design combining immediate release (IR) paracetamol with a second layer of SR paracetamol.

The primary study objective was to compare the analgesic efficacy of a single dose of the SR paracetamol formulation, with that of a standard IR paracetamol formulation, Panadol, for the management of acute post-operative pain. The secondary objectives of the study were to compare the duration of analgesic effect of SR paracetamol with that of IR paracetamol and to evaluate the safety of the two formulations. 


\section{Methods}

\section{Protocol}

This was a multi-centre, single dose, double-blind, randomised, controlled trial conducted in accordance with the Declaration of Helsinki and Good Clinical Practice. ${ }^{10,11}$ The study was conducted at five University Dental Hospitals in the UK and Ireland and approved by the local research ethics committee of each study centre.

Patients who required at least one lower impacted third molar to be removed under general anaesthesia and necessitating bone removal were invited to participate in the study. Patients who had upper and/or contralateral teeth that also required removal were eligible for inclusion.

Patients were informed about the study both verbally and in writing at a screening visit and all patients gave their written, informed consent to participate in the study. A routine dental examination was performed as well as an overall assessment of general health. Demographic details were collected and all current medication was recorded. Female patients of child-bearing potential underwent a pregnancy test which had to prove negative. Patients were free to withdraw from the study at any time and were excluded if they:

- suffered any disease that could affect the action, absorption or disposition of the study medication;

- had a medical or social history suggestive of liver impairment;

- used any medication likely to interfere with the action, absorption or disposition of the study medication;

- had a known or suspected intolerance or hypersensitivity to paracetamol, ibuprofen, tramadol or to any of the anaesthetic agents to be used;

- were females who were pregnant, lactating, attempting to become pregnant or taking hormone replacement therapy;

- had a Body Mass Index ${ }^{12}$ greater than $35 \mathrm{~kg} / \mathrm{m}^{2}$.

On the treatment day (no more than 30 days after screening) female patients underwent another pregnancy test which had to prove negative and details of any concomitant medications and adverse events were collected. Patients were excluded if they had taken any analgesics or tranquillisers within 24 hours of the treatment day or used steroids for one month or more during the preceding year. Removal of the lower impacted third molar was carried out using a routine and standardised procedure and general anaesthetic technique. After surgery, as soon as patients became aware of their surroundings they were required to complete an assessment of pain using a visual analogue scale. When the patient's pain reached moderate or severe intensity they were dosed with one of the two study medications. Patients repeated the pain assessments at 10 minute intervals until they reached moderate/severe intensity. Patients who failed to reach the required pain intensity after 60 minutes were not randomised to treatment.

Subsequent to receiving study medication, patients conducted assessments recording the level of pain intensity and pain relief at time intervals for up to 8 hours $(0.5,1,1.5,2,3,4,5,6,7$ and 8 hours). Patients remained in hospital for up to 4 hours after receiving study medication, in order to complete the 4-hour pain assessment and their first global assessment. They were then provided with a patient booklet to complete the remaining pain assessments (up to 8 hours) and the final global assessment at the end of the study at home. Pain intensity was evaluated using a four-point categorical verbal rating scale (VRS) from none (0) to severe (3) and a $100 \mathrm{~mm}$ visual analogue scale (VAS) labelled 'no pain' at one end and 'unbearable pain' at the opposite end. Relief from starting pain was rated using a five-point VRS from no relief (0) to complete relief (4). Global assessments were rated on a five-point VRS from excellent (0) to poor (4) in response to the question, 'Overall, how would you rate this medication?' At each post-operative pain assessment time point, details of any concomitant medications and adverse events were recorded.
If pain intensity was not reduced and adequate pain relief was not achieved then patients were permitted to take rescue medication, although they were encouraged to wait 45 minutes to allow the study medication time to take effect. Rescue medication was either 400-600 mg ibuprofen, 30-60 mg nefopam, 30-60 mg codeine or 50-100 mg tramadol tablets. In cases of extreme pain, intravenous tramadol was available. Rescue medication was also available on return of pain and the time to rescue medication was recorded. To ensure that the information given to patients regarding the requesting of rescue medication was presented in a standard manner, each site was provided with a bullet-point script to use as an aidemémoire which abstracted key information from the protocol.

For patients who used rescue medication, pain intensity and pain relief scores at the time of taking rescue medication were assigned the 'worst case' response and these values were extrapolated to 8 hours for both intention to treat and per protocol analyses. Similarly, for the 4-hour global evaluation, patients taking rescue medication before 4 hours were assigned a global assessment of 4 (poor), and this value was also carried forward for the overall assessment at the end of the study ( 8 hours). Those who took rescue medication between 4 hours and 8 hours provided an overall evaluation of study medication at the time of re-medication, and this value was used for the overall assessment. Patients who did not take rescue medication provided an overall assessment 8 hours after treatment.

The end of the study for each patient was defined as either the completion of the 8-hour assessment or the time of administration of rescue medication (up to 8 hours). At the end of the study, except if rescue medication had been administered, patients completed an overall global assessment of the study medication. No further pain intensity assessments were to be done by the patient and the patient then received normal medical care.

Within ten days of the surgery, patients returned to the clinic for a follow-up assessment, which included a dental examination and collection of details of any concomitant medications and adverse events.

During the study, adverse events were elicited in a standard manner by asking the patient at the beginning of the treatment day and at the follow-up visit, 'Have you felt unwell, experienced any unusual symptoms, taken any new medications or had any change to your current medication since your last visit?'. In addition, any adverse event occurring during the study that was spontaneously reported either by the patient, clinic staff or the investigator was recorded. Before the patient left the clinic on the treatment day, patients were asked, 'Have you had any undesirable effects since taking the medication?'. Once the patient had left the clinic, adverse events were elicited from patients with the following question in the patient booklet: 'Have you had any undesirable symptoms since leaving the hospital and within 8 hours of your operation?'.

The sample size was based on a published study ${ }^{6}$, with 260 patients per treatment group, to permit the study to have at least $80 \%$ power to show that SR paracetamol was no more than $15 \%$ inferior to IR paracetamol at the 4-hour global assessment when comparing the success rates (of very good or excellent) of the two groups (expected to be 27\%). Assuming that at least 90\% of patients would require analgesia on recovery from anaesthesia and satisfactory completion of post-dosing assessments, it was calculated that a total of 576 patients would need to be enrolled to ensure a sufficient number of evaluable patients. These numbers assumed the use of one-tailed 5\% significance tests for the between treatment comparisons. During the study it was discovered that all 50 patients from one of the centres were ineligible, thus the recruitment target was increased from 576 to 627 .

Peak Pain Intensity Difference (PPID) was generated for each patient by taking the largest value available over time for a patient. Summed Pain Intensity Differences (SPID) were generated for each 
patient by subtracting the pain intensity scores of none (0), mild (1), moderate (2) and severe (3) from the baseline pain assessment and summing the values over the 4-, 6- and 8-hour evaluation periods, weighting for time, respectively. ${ }^{13}$ Summed Pain Analogue Intensity Differences (SPAID) were generated for each patient by subtracting the VAS scores from the baseline pain assessment and summing the values over the 4-, 6- and 8-hour evaluation periods, weighting for time, respectively. ${ }^{13}$

Total Pain Relief (TOTPAR) was generated for each patient by summing the hourly pain relief scores of no relief $(0)$, a little relief (1), some relief (2), a lot of relief (3) and complete relief (4) over 1, 4,6 and 8 hours, weighting for time, respectively ${ }^{13}$.

Peak Pain Relief (PPREL) was generated for each patient by taking the largest value available over time for a patient.

\section{Assignment}

Patients were randomised to receive one of two treatments, SR paracetamol or IR paracetamol after surgery, when the post-operative pain had reached moderate/severe intensity as determined by $30 \mathrm{~mm}$ on a VAS. ${ }^{14}$ The computer-generated randomisation schedule was supplied by the biostatistician and study medication was packed and labelled centrally and so the identification was not known by the study centres.

\section{Blinding}

The study included a double-dummy dosing regimen to maintain the study blinding. This was necessary because the SR paracetamol tablet is larger and heavier than the IR paracetamol tablet.

\section{Study medications}

Study medications were taken with $100 \mathrm{ml}$ of water as below.

\section{SR paracetamol}

Two SR paracetamol tablets and two IR paracetamol placebo tablets.

\section{IR paracetamol}

Two IR paracetamol tablets and two SR paracetamol placebo tablets. SR paracetamol tablets contain $665 \mathrm{mg}$ paracetamol and IR paracetamol tablets contain $500 \mathrm{mg}$ paracetamol. The doses selected for this single dose study were two tablets of IR paraceta$\mathrm{mol}$ (1000 $\mathrm{mg}$ paracetamol) and two tablets of SR paracetamol (1330 mg paracetamol). The reason for the higher paracetamol content in a SR paracetamol tablet compared with a IR paracetamol tablet is to ensure that the new formulation will provide adequate analgesia with a three times daily dose regimen compared with four times daily for IR paracetamol. In a single dose pharmacokinetic study, it has been shown that SR paracetamol is bioequivalent to IR paracetamol with respect to dose-corrected AUC (SB data on file).

\section{Results}

Seven hundred and thirty five patients were screened for this study and 627 of these were randomised to treatment. Figure 1 shows a flow diagram illustrating the disposition of patients in the trial. Two populations were identified for analysis: a per protocol and an intention to treat population. All patients who were randomised, entered the treatment period and took at least one tablet of study medication were considered evaluable for the intention to treat population (total $=627$ patients). These patients also served as the safety population. Patients were considered eligible for the per protocol population if they entered the treatment period and did not violate the protocol in a major way (total $=510$ patients). Protocol violations included: use of prohibited concomitant medications, the patient not remaining in the hospital up to and including the 4 hour assessments or the patient vomiting within the first 2 hours of the assessment period.

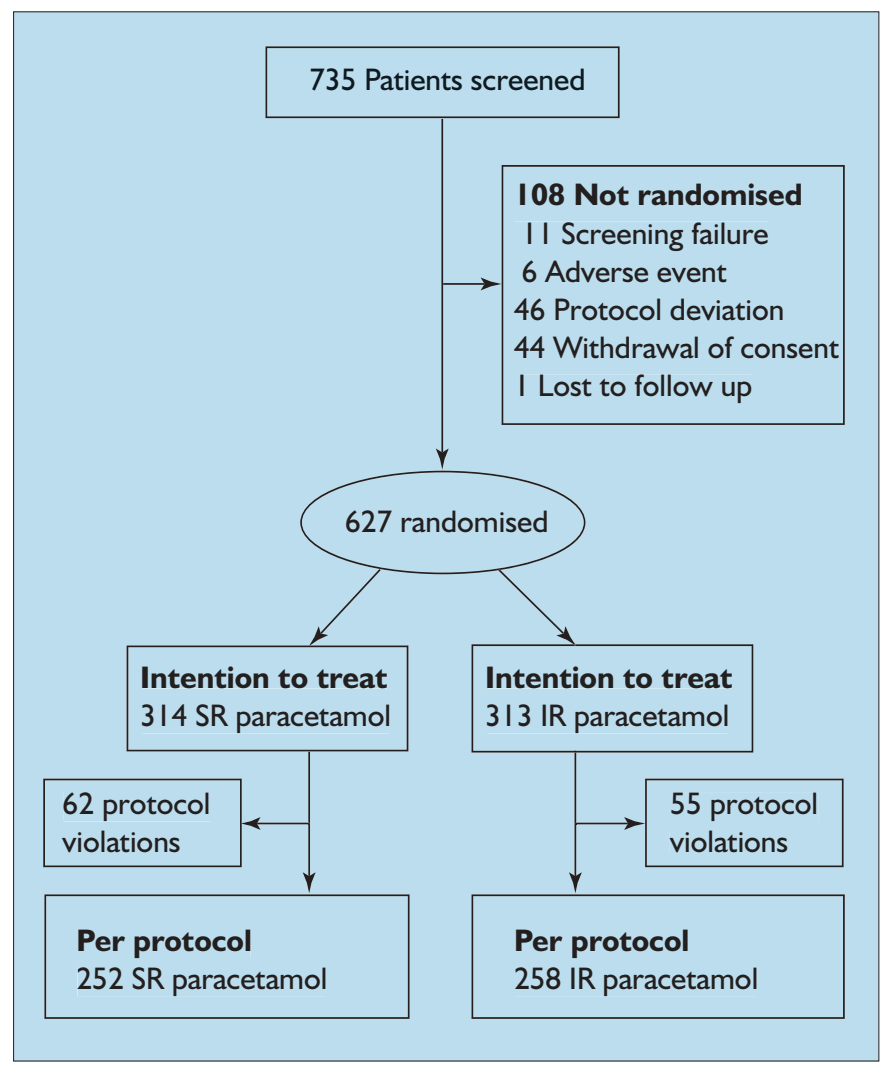

Fig. I Disposition of patients.

The demographic characteristics for the per protocol and intention to treat populations are shown in Table 1. As the primary analysis focused on the per protocol population, the following describes demographic and baseline details for the 252 patients in the SR paracetamol group and the 258 patients in the IR paracetamol group who qualified for the per protocol analysis. On average, three teeth were extracted from patients in both treatment groups and the median duration of surgery was 20 minutes (range 4-60 $\mathrm{min}$ ) for the SR paracetamol group and 17 minutes (range 3-75 $\mathrm{min}$ ) for the IR paracetamol group. These findings were very similar for the intention to treat population.

The baseline vital signs for the per protocol population were very similar for the two groups and are summarised in Table 2.

Table I. Demographic characteristics for the per protocol (PP) and intention to treat (ITT) populations

\begin{tabular}{|c|c|c|c|c|}
\hline & SR paracetam & & IR paracetam & \\
\hline & PP & ITT & PP & ITT \\
\hline Population & 252 & 314 & 258 & 313 \\
\hline Age (years) & & & & \\
\hline Median & 24.9 & 25.2 & 25.4 & 25.3 \\
\hline Minimum & 18.3 & 18.3 & 18.1 & 18.1 \\
\hline Maximum & 45.0 & 45.0 & 51.5 & 51.5 \\
\hline Sex & & & & \\
\hline Male & $77(30.6 \%)$ & $100(31.8 \%)$ & $84(32.6 \%)$ & 105 (33.5\%) \\
\hline Female & 175 (69.4\%) & $214(68.2 \%)$ & $174(67.4 \%)$ & $208(66.5 \%)$ \\
\hline Race & & & & \\
\hline Caucasian & 230 (91.3\%) & 280 (89.2\%) & 233 (90.3\%) & $28 \mathrm{I}(89.8 \%)$ \\
\hline Black & $9(3.6 \%)$ & $13(4.1 \%)$ & II (4.3\%) & $14(4.5 \%)$ \\
\hline Asian & $10(4.0 \%)$ & $16(5.1 \%)$ & $12(4.7 \%)$ & 15 (4.8\%) \\
\hline Other & $3(1.2 \%)$ & $5(1.6 \%)$ & $2(0.8 \%)$ & $3(1.0 \%)$ \\
\hline Body Mass I & $\mathrm{x}\left(\mathrm{kg} / \mathrm{m}^{2}\right)$ & & & \\
\hline Mean & 23.5 & 23.5 & 23.6 & 23.7 \\
\hline Minimum & 16.8 & 16.8 & 16.1 & 16.1 \\
\hline Maximum & 34.0 & 34.0 & 34.5 & 34.5 \\
\hline
\end{tabular}


Table 2 Summary of baseline vital signs (per protocol population)

\begin{tabular}{lll}
\hline & \multicolumn{2}{c}{ Median value } \\
& $\begin{array}{l}\text { SR paracetamol } \\
(\mathrm{n}=252)\end{array}$ & $\begin{array}{l}\text { IR paracetamol } \\
(\mathrm{n}=258)\end{array}$ \\
\hline Systolic blood pressure & $120 \mathrm{mmHg}$ & $120 \mathrm{mmHg}$ \\
Diastolic blood pressure & $73.5 \mathrm{mmHg}$ & $72 \mathrm{mmHg}$ \\
Heart rate & $73 \mathrm{bpm}$ & $76 \mathrm{bpm}$ \\
Respiratory rate & $18 \mathrm{rpm}$ & $18 \mathrm{rpm}$ \\
Temperature & $36.5^{\circ} \mathrm{C}$ & $36.5^{\circ} \mathrm{C}$ \\
\hline
\end{tabular}

\section{Primary efficacy parameter}

The primary measure of analgesic efficacy was the patients' global evaluation at 4 hours. Patients that took rescue medication before the 4-hour evaluation were assigned a response of poor. Figure 2 shows the number and percentage of patients by response to each treatment whilst Table 3 shows the response rates classified as successes and failures, along with the results of the statistical analysis. A successful response was defined as a very good or excellent global assessment 4 hours after drug administration ${ }^{6}$. The difference in the proportion of success between the two treatments and the $90 \%$ confidence interval for this difference are shown in Table 3. The pre-specified critical value for the lower bound of the $90 \%$ confidence interval, which determined whether SR paracetamol was inferior to IR paracetamol, was $-15 \%$. The observed value of $+0.6 \%$ indicates that the two treatments can be concluded to be equivalent with respect to the global assessment at 4 hours.

In order to take account of the duration of surgery (not significant), the number of teeth removed (not significant) and the effects of centre differences (highly significant; differences were seen between the centres in global pain relief at 4 hours), an odds ratio adjusted for these factors was also calculated. The critical value for the lower bound of the $90 \%$ confidence interval for the odds ratio, which determined whether SR paracetamol was inferior to IR paracetamol, was 0.38 . The observed value of 0.91 indicates SR paracetamol is not inferior to IR paracetamol with respect to the global assessment at 4 hours. The treatment by centre interaction was investigated and found to be insignificant for the per protocol analysis, hence the results are presented over all centres. This confirms the findings from the unadjusted analysis.

\section{Secondary efficacy parameters: time to re-medication}

No statistically significant differences were seen in the times to rescue medication between the two treatment groups. However, the estimated median time to re-medication was longer for the SR paracetamol group ( $4 \mathrm{hr} 5 \mathrm{~min}$ ) compared with the IR paracetamol group ( $3 \mathrm{hr} 10 \mathrm{~min}$ ). At the end of the study period ( 8 hours), approximately $20 \%$ of patients in both groups had not taken any rescue medication.

\section{Secondary efficacy parameters: pain intensity}

There was no evidence of any statistically significant differences between the two treatment groups for PPID. Based on the fourpoint VRS data, the median PPID was 1.00 for both treatments indicating that SR paracetamol and IR paracetamol provide a similar maximum level of analgesia. No statistically significant differ-

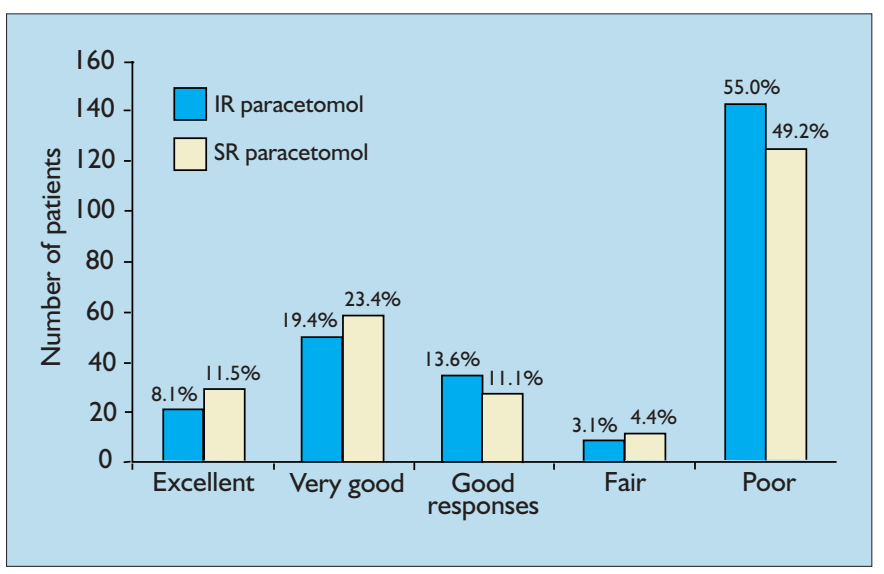

Fig. 2 Summary statistics for global evaluation at $\mathbf{4}$ hours for the per protocol population. There were three missing global assessments: two patients were withdrawn due to adverse events and the 4-hour assessment was not collected for one patient.

ences were seen in the time to PPID, suggesting that the treatments take a similar length of time to reach peak effect. The median time to PPID for both groups was $1 \mathrm{hr} 30 \mathrm{~min}$.

There was no evidence of any statistically significant differences between the two treatment groups when the SPID, based on the four-point VRS analysis, at any of the time points although there was a trend in favour of SR paracetamol. However, SR paracetamol was statistically significantly better than IR paracetamol when the SPAID were analysed at the 6-hour $(\mathrm{p}=0.0344)$ and 8-hour $(\mathrm{p}=0.05)$ assessments. Significant differences in favour of SR paracetamol at the 6- and 8-hour assessments were found for both SPID ( $\mathrm{p}=0.0247$ and $\mathrm{p}=0.0327$, respectively) and SPAID ( $p=0.0095$ and $p=0.0150$, respectively) in the intention to treat analysis. A significant difference was also found for SPAID at 4 hours $(\mathrm{p}=0.0279)$ in the intention to treat analysis.

\section{Secondary efficacy parameters: pain relief}

There was no evidence of any statistically significant differences between the two treatment groups for TOTPAR at any time point or for PPREL. When compared at the earliest time point, 1 hour after administration, the difference between the two treatment groups was very small: the mean TOTPAR for SR paracetamol was 1.32 (Standard Deviation $[\mathrm{SD}]=0.801)$ compared with $1.30(\mathrm{SD}=0.849)$ for IR paracetamol. At the later time points progressively greater differences were observed between the formulations. For example, at 8 hours the mean TOTPAR for SR paracetamol was $10.80(\mathrm{SD}=8.166)$ compared with $9.80(\mathrm{SD}=7.193)$ for IR paracetamol. The mean PPREL for SR paracetamol $(2.46, \mathrm{SD}=1.17)$ was almost identical to that for IR paracetamol $(2.45, \mathrm{SD}=1.15)$ suggesting that the maximum level of the analgesic response provided by both treatments is very similar. These two values approximate to a rating of between 'some relief' and 'a lot of relief' on the five-point VRS of pain.

No statistically significant differences were seen in the time to PPREL between SR paracetamol and IR paracetamol. The median time to PPREL for both groups was $1 \mathrm{hr} 30 \mathrm{~min}$ and the confidence intervals of these medians were identical.

Table 3 Global evaluation: results unadjusted for the duration of surgery, number of teeth removed or centre for the per protocol population

\begin{tabular}{lccc}
\hline Rating & $\begin{array}{l}\text { Response rates } \\
\text { SR paracetamol }(\mathrm{n}=252)\end{array}$ & IR paracetamol $(\mathrm{n}=258)$ & $\begin{array}{l}\text { Estimated treatment difference: } \\
\text { SR paracetamol - IR paracetamol }\end{array}$ \\
\hline Very good/excellent & $88(35.1 \%)$ & $71(27.7 \%)$ & $7.3 \%$ \\
Poor/fair/good & $163(64.9 \%)$ & $185(72.3 \%)$ & \\
\hline
\end{tabular}

* Analysis does not include three missing cases 
Table 4 Summary of the ten most frequently reported treatment emergent adverse events

\begin{tabular}{lllc}
\hline Adverse event & $\begin{array}{l}\text { Total number of } \\
\text { adverse events } \\
(\mathrm{n}=604)\end{array}$ & $\begin{array}{l}\text { Total number of patients with adverse } \\
\text { events }(\%) \\
\text { SR paracetamol }\end{array}$ & IR paracetamol \\
\hline Nausea & 74 & $166(52.9 \%)$ & $163(52.1 \%)$ \\
Vomiting & 58 & $29(9.2 \%)$ & $37(11.8 \%)$ \\
Dystonia & 45 & $22(7.0 \%)$ & $22(7.0 \%)$ \\
Hypesthesia & 39 & $24(7.6 \%)$ & $21(6.7 \%)$ \\
Paraesthesia & 38 & $17(5.4 \%)$ & $17(5.4 \%)$ \\
Dizziness & 38 & $19(6.1 \%)$ & $17(5.4 \%)$ \\
Face oedema & 38 & $10(3.2 \%)$ & $25(8.0 \%)$ \\
Headache & 33 & $22(7.0 \%)$ & $14(4.5 \%)$ \\
Infection & 31 & $16(5.1 \%)$ & $16(5.1 \%)$ \\
Haemorrhage & 30 & $22(7.0 \%)$ & $9(2.9 \%)$ \\
\hline
\end{tabular}

\section{Secondary efficacy parameters: overall assessment at the end of} the study

Forty-two (23\%) patients treated with SR paracetamol reported a very good or excellent response at 8 hours (or between 4-8 hours if they took rescue medication), whilst 34 (17.3\%) of the IR paracetamol treated patients reported such a response.

\section{Efficacy results: intention to treat population}

The results of the per protocol analysis and the intention to treat analysis were very similar other than those described above.

\section{Safety evaluation}

A total of 604 adverse events emerged during the treatment period from a safety population of 627 patients. Of the 314 patients randomised to SR paracetamol, 166 (52.9\%) reported 321 adverse events. Of the 313 patients randomised to IR paracetamol, 163 $(52.1 \%)$ reported 283 adverse events. Most of the adverse events were considered unrelated to study medication and Table 4 includes information on the ten most frequent reported events by treatment group.

Three patients each reported a single serious adverse event (postoperative haemorrhage, hypotension and acute post-operative wound infection). None was related to study medication and all resolved without complication.

\section{Discussion}

\section{Primary efficacy parameter}

In the management of acute post-operative pain following the surgical removal of wisdom teeth, a single dose of SR paracetamol was shown to be clinically and statistically equivalent to IR paracetamol measured by the patients' global response at 4 hours after administration. Paracetamol is recommended for mild to moderate pain ${ }^{15}$. However, the pain intensity associated with removal of impacted third molars is moderate to severe ${ }^{16}$, therefore the present study has provided a stringent test for SR paracetamol and IR paracetamol.

\section{Secondary parameters}

The mean PPREL for patients treated with SR paracetamol was almost identical to that for IR paracetamol, based on the per protocol analysis, suggesting that the magnitude of the analgesic response provided by both treatments was very similar. This result is supported by the analysis of the median PPID. The median time taken to reach PPREL and PPID was $1 \mathrm{hr} 30 \mathrm{~min}$ for both SR paracetamol and IR paracetamol, suggesting that the development of analgesia was similar for both formulations. The observations that there was no significant difference in TOTPAR between SR paracetamol and IR paracetamol measured 1 hour after administration of study medication is also consistent with this conclusion, although SR paracetamol provided progressively greater benefit than IR paracetamol over time. Furthermore, analysis of the intention to treat population was supportive.

Trends in favour of SR paracetamol were observed among the secondary parameters and these tended to emerge at the later time points. This was apparent for both the per protocol and intention to treat populations. For example, while there were no statistically significant differences in the time to re-medication between the groups, the median time to re-medication was longer for patients treated with SR paracetamol compared with IR paracetamol, based on the per protocol population. Time to re-medication provides an indication of the duration of analgesia. The high re-medication rate observed is common in studies of post-operative dental pain ${ }^{5}$ and typically about $50 \%$ of patients re-medicate within 4 hours after treatment with $1000 \mathrm{mg}$ IR-paracetamol ${ }^{4,6}$.

Significant differences in favour of SR paracetamol were also apparent in certain of the summed pain measurements. For example, SPAID, based on the VAS for the per protocol population, showed that SR paracetamol was statistically significantly better than IR paracetamol at both the 6- and 8-hour assessments. Significance in favour of SR paracetamol was also confirmed in the intention to treat analysis. Whilst there were no significant differences at any time point for SPID, based on the VRS for the per protocol population, there was a trend in favour of SR paracetamol. The significant differences in favour of SR paracetamol found for SPID in the intention to treat analysis supports this trend. These differences are as expected given that SR paracetamol contains sustained release paracetamol and each tablet contains $665 \mathrm{mg}$ paracetamol compared with $500 \mathrm{mg}$ for IR paracetamol. The VAS provides a more sensitive and accurate representation of pain intensity than a descriptive pain scale ${ }^{17}$. This may explain why a statistical significance was noted for SPAID and not for SPID.

Area under the curve measures such as TOTPAR and SPID confound onset and duration of analgesia with peak effect. Since SR paracetamol and IR paracetamol were similar with respect to the development of analgesia and the peak analgesic effect, the fact that SPAID revealed significant differences in favour of SR paracetamol at the 6-and 8-hour assessments suggests that the duration of activity of SR paracetamol is longer than IR paracetamol. This interpretation is in accordance with the observation that median time to re-medication was longer in patients treated with SR paracetamol compared with IR paracetamol and as expected for a formulation containing sustained release paracetamol.

A total of five centres participated in this study. Treatment differences between SR paracetamol and IR paracetamol were not significant across the centres in the per protocol population, but this interaction proved to be significant for the intention to treat population. Data generated at one of the centres in the intention to treat analysis showed a trend in favour of IR paracetamol whereas at all other centres the trend was in favour of SR paracetamol. The likely reason this did not have an impact on the per protocol analysis is that all patients at this centre were excluded from this analysis because they received Maxolon (metoclopramide), a prohibited medication, prior to surgery. Metoclopramide acts on the upper gastrointestinal tract to prevent nausea and vomiting ${ }^{15}$, which can occur following general anaesthesia. It would therefore enhance the rate of absorption of a drug such as paracetamol and thus the onset of analgesia. While it is possible that use of metoclopramide at this centre may explain why treatment difference between SR paracetamol and IR paracetamol is reversed, this conclusion remains speculative.

\section{Safety evaluation}

This was a large study with 627 patients randomised and treated with the study medication thus constituting a large safety population. Whilst there were a large number of adverse events reported 
in this study, most of these were considered by the investigators to be unrelated to the study medication. Such events are likely to have resulted from the surgery and/or general anaesthesia, for example, nausea, vomiting and dizziness. However, it is not possible to distinguish these from any that may have been caused by a single dose of analgesic provided.

\section{Conclusions}

Based on the primary parameter (4-hour global assessment) it is concluded that SR paracetamol and IR paracetamol are clinically and statistically equivalent. SR paracetamol and IR paracetamol were found to be similar in terms of both development of analgesia and peak analgesic affect. SR paracetamol had a longer duration of activity than IR paracetamol as demonstrated by statistically significant differences in SPAID at 6 and 8 hours and supported by a longer time to re-medication. This is as expected from a formulation containing SR paracetamol and a higher dose of paracetamol. The safety profiles of SR paracetamol and IR paracetamol were very similar based on the results from this single dose study in post-operative dental pain.

The authors wish to acknowledge the assistance of study co-ordinators, Debbie Jackson-Leech (Manchester), Rita Walker (Cardiff), Conor Barry (Dublin) and Lindsay Stenhouse (Birmingham) and also that of Michelle Estruch and Mairead Moohan (GlaxoSmithKline Consumer Healthcare) for their statistical input into this paper. We also wish to acknowledge the support of GlaxoSmithKline Consumer Healthcare which enabled this study to be carried out.

1 Moore A, Collins S, Carroll D, McQuay H, Edwards J. Single dose paracetamol (acetaminophen), with and without codeine, for postoperative pain. Cochrane Database Syst Rev. 2000;(2):CD001547.

2 Rang H P, Dale M M, Ritter J M. Anti-inflammatory and immunosuppresant drugs. In: Pharmacology Fourth Edition. pp229-247. Edinburgh: Churchill Livingstone, 1999.

3 Bentley K C, Head T W. The additive analgesic efficacy of acetaminophen $1000 \mathrm{mg}$, and codeine, $60 \mathrm{mg}$, in dental pain. Clin Pharmacol Ther 1987; 42: 634-640.
4 Kiersch T A, Halladay S C, Hormel P C. A single-dose, double-blind comparison of naproxen sodium, acetaminophen and placebo in postoperative dental pain. Clin Ther 1994; 16: 394-404.

5 Mehlisch D R, Sollecito W A, Helfrick J F et al. Multicenter clinical trial of ibuprofen and acetaminophen in the treatment of postoperative dental pain. J Am Dent Assoc 1990; 121: 257-263.

6 Cooper S A, Schachtel P, Goldman E, Gelb S, Cohn P. Ibuprofen and acetaminohen in the relief of acute pain: A randomised, double-blind placebo-controlled study. J Clin Pharmacol 1989; 29: 1026-1030.

7 Nguyen A M, Graham D Y, Gage T, Griffiths G R. Nonsteroidal antiinflammatory drug use in dentistry: gastrointestinal implications. Gen Dent 1999; 47:590-596.

8 McQuay H, Moore A. An evidence based resource for pain. pp187-192. Oxford: Oxford University Press, 1998.

9 British Medical Association and Royal Pharmaceutical Society of Great Britain. British National Formulary Volume 39. Oxon: Pharmaceutical Press, 2000: 205-208.

10 Recommendations Guiding Physicians in Biomedical Research Involving Human Subjects. Adopted by the 18th World Assembly, Helsinki, Finland, June 1964, amended by the 29th World Medical Assembly, Tokyo, Japan, October 1975, the 35th World Medical Assembly, Hong Kong, September 1989 and the 48th General Assembly, South Africa, October 1996.

11 ICH Topic 6 Guideline for Good Clinical Practice CPMP/ICH/135/95 17 July 1996.

12 Pengally C D R. Body Mass Index and abdominal girth in the diagnosis of obesity. Proc R Coll Physicians Edinb 1994; 24: 174-180.

13 Max M B, Laska E M. Single-dose analgesic comparisons. In: Advances in Pain Research and Therapy, Vol 18: 55-95. The Design of Analgesic Clinical Trials. Eds. Max M B, Portenoy R K, Laska E M (eds). New York, Raven Press, 1991.

14 Collins S L, Moore A, McQuay H J. The visual analogue pain intensity scale: what is moderate pain in millimetres? Pain. 1997; 72: 95-97.

15 Dollery C T (ed). Therapeutic Drugs. Vol. 2: M132-M136. Edinburgh: Churchill Livingstone, 1999.

16 Cooper S A. Models for clinical assessment of oral analgesics. Am J Med 1983; 75: 24-29.

17 Sriwatanakul K, Kelvie W, Lasagna L, Calimlim J F, Weis O F, Mehta G. Studies with different types of visual analog scales for measurement of pain. Clin Pharmacol Ther 1985; 34: 234-239. 